\title{
Ischemic Colitis of the Left Colon in a Diabetic Patient
}

\author{
Anastasios J. Karayiannakis ${ }^{a}$ Helen Bolanaki \\ Georgios Kouklakis $^{\mathrm{b}}$ Konstantinos Dimakis ${ }^{\mathrm{a}} \quad$ Ilker Memet $^{\mathrm{a}}$ \\ Constantinos Simopoulos ${ }^{\mathrm{a}}$
}

aSecond Department of Surgery and bastrointestinal Endoscopy Unit, Democritus University of Thrace, Medical School, Alexandroupolis, Greece

\section{Key Words}

Ischemic colitis · Diabetes mellitus · Complications

\begin{abstract}
Diabetes mellitus may affect the gastrointestinal tract possibly as a result of autonomic neuropathy. Here we present a 68-year-old male with non-insulin-dependent diabetes mellitus who presented with prolonged watery diarrhea and in whom imaging studies demonstrated ischemic colitis of the left colon. Resection of the affected colon resulted in sustained disappearance of symptoms.
\end{abstract}

\section{Introduction}

Diabetes mellitus is the most common endocrine disorder affecting multiple organs including the gastrointestinal (GI) tract where manifestations and/or complications relate to disordered gut motility possibly as a result of autonomic neuropathy. Here we present a case of ischemic colitis of the left colon in a patient with non-insulin-dependent diabetes.

\section{Case Report}

A 68-year-old male presented with a 4-month history of colicky abdominal pain and watery diarrhea (15-18 large-volume bowel movements daily) containing excess of mucus but not blood. The pain was frequently accompanied by defecation urgency and was in part relieved by bowel movements.

His past medical history included cerebral infarction at the left temporal area 8 years before without any residual neurologic symptoms and non-insulin-dependent diabetes mellitus treated with glipizide ( $5 \mathrm{mg}$, three times daily) maintaining serum glucose level below $150 \mathrm{mg} / \mathrm{dl}$. Glycemic control was based on day-to-day blood glucose monitoring by using an at-home glucometer. Glycated hemoglobin (HbAlc) testing was performed every 3 months with medication and diet adjustments aiming at a $\mathrm{HbAlc}<7 \%$ for optimal glycemic control. He had also mild hypertension of the same duration treated

Anastasios J. Karayiannakis, $\mathrm{MD}, \mathrm{MSc}, \mathrm{PhD}$
Second Department of Surgery, Democritus University of Thrace, Medical School GR-68100 Alexandroupolis (Greece)

Tel. +30 25510 74006, E-Mail akarayan@usa.net 
with calcium ion channels blocker (felodipine, $5 \mathrm{mg}$ daily) and $\beta$-blocker (metoprolol fumarate, $47.5 \mathrm{mg}$ daily).

On physical examination the abdomen was mildly distended and a $12-\mathrm{cm}$-long, firm mass with mild tenderness was palpable in the lower abdomen below the umbilicus. There was no guarding or rebound tenderness and bowel sounds were normal on auscultation. Serum glucose level was $163 \mathrm{mg} / \mathrm{dl}$ with the rest of routine laboratory examinations and tumor markers being normal. Multiple stool cultures were negative, failing to detect any parasites or bacteria responsible for the diarrhea. Colonoscopy showed extensive mucosal edema and hyperemia, with narrowing of the bowel lumen extending $20 \mathrm{~cm}$ from the anal verge up to the splenic flexure. Random endoscopic biopsies showed capillary basement membrane thickening in the submucosal arterioles and submucosal edema. A double contrast barium enema showed a serrated contour with typical thumbprinting, loss of colonic haustra, rigidity and narrowing of the lumen of the descending and sigmoid colon, which did not resolve after intravenous glucagon infusion or intraluminal air insufflation (fig. 1). Computed tomography (CT) demonstrated a markedly thickened bowel wall in the sigmoid colon and an edematous sigmoid mesocolon (fig. 2).

Exploratory laparotomy was undertaken with the presumptive diagnosis of ischemic colitis. The small bowel and the colon proximal to the splenic flexure appeared entirely normal. The left colon from the splenic flexure up to the peritoneal reflection was edematous with a markedly thickened wall. The sigmoid mesocolon was also extensively thickened and fibrotic. The inferior mesenteric artery was dissected and found to have palpable pulse. The entire left colon was resected and an end-to-end anastomosis was performed between the transverse colon and the rectum.

The macroscopic appearance of the resected specimen included edematous and thickened colonic mucosa and bowel wall. Microscopic examination showed fibrosis of the entire colonic wall especially in the submucosal layer. Mucosal necrosis, submucosal edema with focal hemorrhage and full-thickness inflammatory cell infiltration were also observed. Thick-walled blood vessels of small caliber with luminal narrowing were found in the lamina propria of the bowel wall and in the sigmoid mesocolon.

The patient had an uneventful postoperative course with disappearance of diarrhea. Funduscopic examination, neurologic evaluation and urinary albumin excretion rate estimation revealed mild diabetic retinopathy but no neuropathy or nephropathy. The patient remains well without any abdominal symptoms 63 months after his operation.

\section{Discussion}

Diabetes mellitus is a systemic disease affecting the function and structure of many organs including the GI tract. An increased prevalence of GI symptoms or complications has been documented in diabetic patients compared with nondiabetic control subjects including symptoms from both the upper and lower GI tract such as gastroparesis, anorexia, vomiting, early satiety, intestinal enteropathy, diarrhea, constipation or fecal incontinence [1-5]. These symptoms are usually attributed to altered GI motility as a result of diabetic autonomic neuropathy involving the GI tract [1]. However, the duration of diabetes [5], poor glycemic control [2-4], the type of diabetes treatment [6], and the presence of diabetic complications, especially peripheral neuropathy [3], may affect the frequency and magnitude of GI complications. The prevalence of diarrhea in diabetic patients varies between 8 and 22\% [1,6-8] and consists of chronic intermittent episodes of voluminous, watery stools for days or months alternated with normal bowel movements or even constipation. It occurs more frequently among type I diabetic patients compared with type II diabetes (non-insulin-dependent) patients and is associated with long-standing poorly controlled disease and the presence of autonomic neuropathy. The diarrhea is usually severe (more than 10 bowel movements per day), explosive, often exacerbates at night and occasionally associates with fecal incontinence [6]. 
The pathogenesis of diarrhea is complex and multifactorial. Alterations in the intestinal motility due to autonomic neuropathy and small bowel bacterial overgrowth are the main causative factors, although medications altering intestinal motility and absorption such as metformin, exocrine pancreatic insufficiency, steatorrhea and malabsorption of bile salts, celiac disease and anorectal dysfunction may also contribute to diabetic diarrhea [1, 6-8]. Infection with Clostridium difficile may also contribute to diarrhea especially in hospitalized patients. Diabetes along with hypoalbuminemia are both significant risk factors for $C$. difficile infection recurrence. Their effect is synergistic, conferring a more than eight-fold increase in odds for recurrence with diabetes being a significant independent risk factor (adjusted odds ratio (AOR) ranging from 3.79 to 5.46) for recurrent C. difficile infection [9]. We presume that this patient, in whom repeated testing for $C$. difficile infection was negative, was not suffering from chronic diabetic diarrhea not only because its features did not resemble those of diabetic diarrhea, but mainly because of the permanent, long-term disappearance of diarrhea after surgery. Instead, the diarrhea should be attributed to segmental ischemic colitis of the left colon. This condition has been reported only occasionally [10-12]. Sharieff et al. [11] reported the case of a 65-year-old man with diabetes mellitus of new onset complicated by nonketotic hyperosmolar coma who developed acute mesenteric ischemia secondary to severe dehydration and hyperosmolarity and who required emergency surgery for infarction of the terminal ileum, the ascending and the transverse colon. Similarly, surgery was necessary because of necrosis of the bowel wall in a 29-year-old woman with insulin-dependent diabetes who was diagnosed as having ischemic colitis of the transverse colon secondary to diabetic angiopathy [10]. Finally, Nagai et al. [12] presented a 70-year-old man suffering from non-insulin-dependent diabetes complicated by nonocclusive colonic ischemia because of altered mesenteric microcirculation and a hypercoagulable state who was treated conservatively by vasodilator infusion and anticoagulant therapy.

Ischemic colitis is the result of nonocclusive mesenteric ischemia in which, unlike occlusive ischemia after arterial embolism and arterial or venous thrombosis, there is time for intestinal collateral flow to develop, thus preventing full-thickness necrosis of the bowel wall. The condition usually involves the left colon near the splenic flexure because of incomplete collaterals between the superior and inferior mesenteric arteries, although right colonic involvement has been described in younger patients and has been associated with a particularly poor prognosis [13]. Ischemic colitis affects mostly elderly people and can be seen after major cardiovascular surgery, in low-flow states such as congestive heart failure, arrhythmia, hypotension and drug-induced vasoconstriction, or may accompany systemic disorders like coagulopathies or rheumatic diseases. Among numerous diseases and medications associated with the development of ischemic colitis, an increased independent correlation was reported not only for diabetes (AOR: 1.82, 95\% confidence interval (CI): 1.31-2.53), but also for hypertension (AOR: 3.21, 95\% CI: 2.28-4.53), chronic obstructive pulmonary disease (AOR: 3.13 , 95\% CI: $2.06-4.75$ ), atrial fibrillation (AOR: 2.21, 95\% CI: 1.34-3.64), congestive heart failure (AOR: 1.94, 95\% CI: 1.11-3.39), antibiotics (AOR: 3.30, 95\% CI: 2.19-4.96), opioids (AOR: 1.96, 95\% CI: 1.43-2.67) and potentially constipating drugs (AOR: 1.75, 95\% CI: 1.25-2.44) [14]. When cardiovascular risk factors, cardiovascular diseases and associated treatments were specifically evaluated for their relationship to the development of colonic ischemia in a cohort of patients requiring admission due to an episode of ischemic colitis, diabetes mellitus (odds ratio (OR): 1.76, 95\% CI: 1.001-3.077), dyslipidemia (OR: 2.12, 95\% CI: 1.26-3.57), heart 
failure (OR: 3.17, 95\% CI: 1.31-7.68), peripheral arterial disease (OR: 4.1, 95\% CI: 1.32-12.72), and treatment with digoxin (OR: $0.27,95 \%$ CI: $0.084-0.857$ ) or acetylsalicylic acid (OR: $1.97,95 \%$ CI: 1.16-3.36) were found to be independently associated with the development of ischemic colitis [15]. In diabetic patients, there is subepithelial collagenous thickening of colorectal mucosa along with basal membrane thickening and luminal narrowing in capillary vessels of the GI tract $[16,17]$. These morphologic changes besides autonomic neuropathy-induced functional abnormalities in the mesenteric circulation may affect microvascular hemodynamics with deterioration of mesenteric microcirculation and development of colonic ischemia in the absence of major vessel occlusion. Diabetes mellitus often associates with hypertension as in our patient. Hypertension-related capillary basement membrane thickening of small vessels and atherosclerotic narrowing of main visceral arteries may further aggravate mesenteric ischemia in diabetics [13]. Ischemia of the large bowel produces a spectrum of pathologic changes consistent with acute on chronic mesenteric ischemia. Depending on the stage at which the tissue is examined, findings may reflect an acute injury mimicking acute Crohn's disease [18] or a more subacute or chronic process where mucosal atrophy and granulation tissue may resemble $C$. difficile colitis. Lamina propria hemorrhage, full-thickness mucosal necrosis and scattered distribution of pseudomembranes are more common in ischemic rather than $C$. difficile colitis. The presence of a hyalinized lamina propria appears to be a specific marker for ischemia whereas the presence of atrophic microcrypts, although not specific, is characteristic of ischemic colitis [19]. The combination of these two features may differentiate ischemic colitis from other colitides, including $C$. difficile colitis. The endoscopic appearance of a focal process and the presence of polyps or masses secondary to submucosal edema within the ischemic areas, known as 'thumbprinting' when detected radiographically, provide further evidence of ischemic colitis.

The clinical presentation of ischemic colitis is nonspecific and its severity depends on the extent of ischemia. Most cases are mild with subacute onset; the ischemic process is transient and limited to the mucosa with fibrosis and formation of colonic strictures in the late phase. However, severe forms with colonic wall necrosis and sepsis have been described $[10,11]$. Abdominal pain often of colicky character, diarrhea and hematochezia have been suggested as common symptoms $[13,20]$. However, diarrhea and hematochezia were absent in previous reports [10-12] whereas our patient had abdominal pain and watery diarrhea only.

Radiological imaging may be helpful in diagnosing ischemic colitis. Barium enema may reveal findings suggestive of mesenteric ischemia such as pneumatosis intestinalis or thumbprinting due to submucosal edema or hemorrhage, ulcerations, segmental strictures and/or dilatation $[10,13,20]$. CT is particularly useful in the acute setting when bowel wall necrosis and peritonitis are suspected by detecting signs which are specific for bowel ischemia such as gas in the bowel wall or portal venous system, whereas in subacute or chronic cases thickening of the bowel wall is a common finding $[11,13]$. Colonoscopy may reveal narrowing of the bowel lumen with pale, edematous mucosa sometimes with petechial hemorrhage $[10,13]$. Colonoscopy either alone or in combination with CT was able to establish the correct diagnosis in 90 and $95 \%$ of cases, respectively, thus, making the use of other diagnostic procedures redundant in the majority of cases [13]. Angiography is rarely helpful because colonic ischemia occurs at the microcirculation level in the absence of occlusion in major vessels $[12,13]$ as in our case where good 
pulsation of the inferior mesenteric artery was palpated intraoperatively, supporting the diagnosis of nonocclusive ischemic colitis.

Treatment of ischemic colitis depends on its severity, with more than half of patients having a moderate form of disease, $34 \%$ of them suffering from severe disease, and in about $10 \%$ of cases there are late ischemic colonic strictures [13]. Conservative therapy, including bowel rest, fluid replacement, administration of antibiotics, vasodilators, oxygen radical scavengers or oxygen radical synthesis inhibitors and anticoagulants, is sufficient for the majority of patients with ischemic colitis due to nonocclusive mesenteric ischemia [13]. Surgery is indicated when bowel wall necrosis and/or peritonitis is suspected, in patients not responding to medical treatment or in those with chronic symptomatic colitis or colonic stricture. Resection of the affected segment with primary anastomosis or transient stoma in the presence of peritonitis is indicated.

In conclusion, ischemic colitis should be considered in diabetic patients having GI symptoms, especially in the setting of diarrhea, and should be promptly evaluated and treated appropriately.
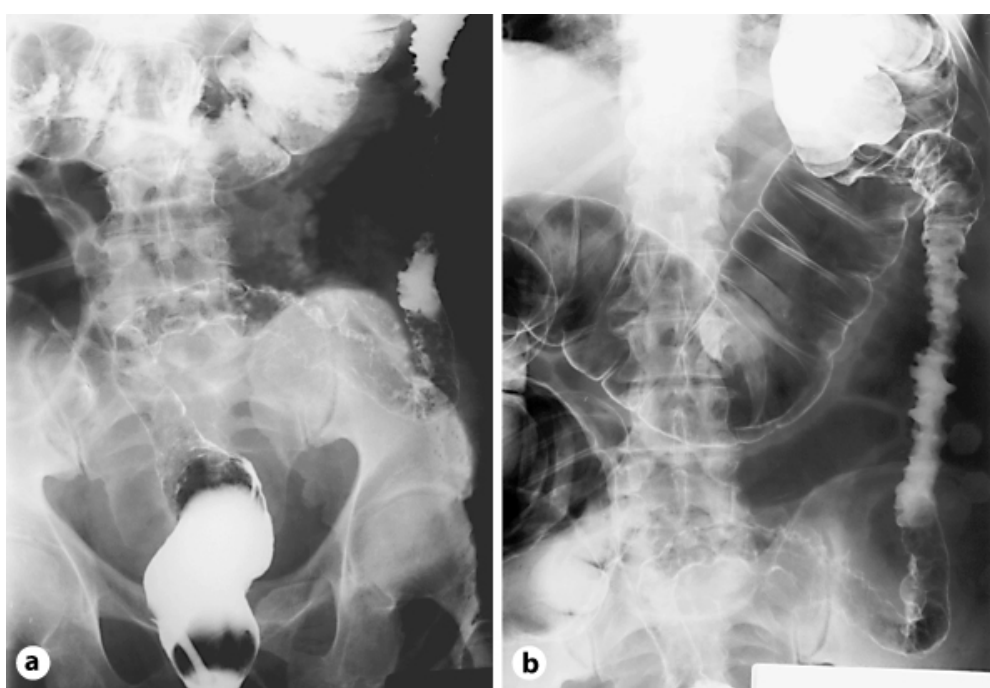

Fig. 1. Barium enema showing stenosis and thumbprinting of the left colon with mild proximal (transverse colon) dilatation. 


\begin{tabular}{r|l|l|l} 
Case Reports in & $\begin{array}{l}\text { Case Rep Gastroenterol 2011;5:239-245 } \\
\text { DOI: 10.1159/000327981 }\end{array}$ & $\begin{array}{l}\text { Published online: } \\
\text { April 15, 2011 }\end{array}$ & $\begin{array}{l}\text { (2011 S. Karger AG, Basel } \\
\text { ISSN 1662-0631 } \\
\text { www.karger.com/crg }\end{array}$ \\
\hline
\end{tabular}

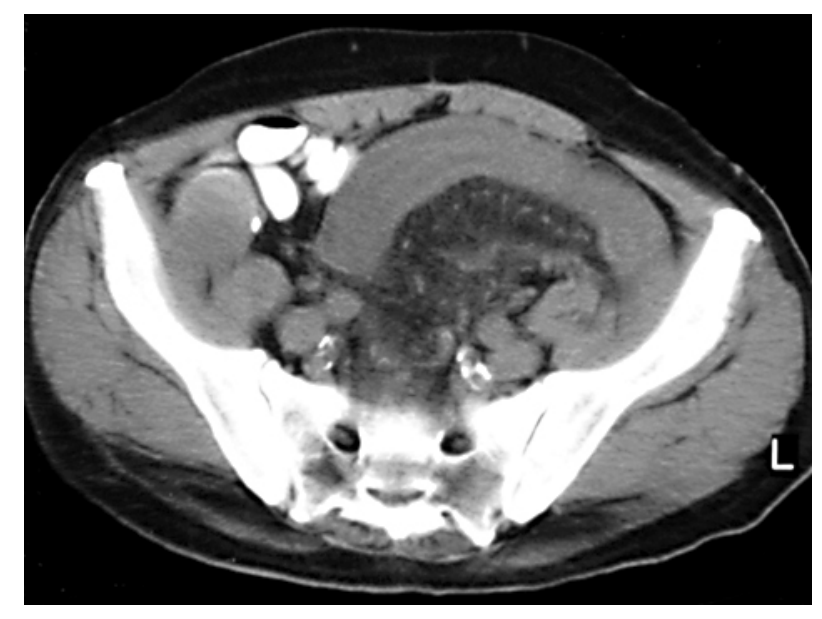

Fig. 2. CT showing marked wall thickening of the sigmoid colon.

\section{References}

1 Feldman M, Schiller LR: Disorders of gastrointestinal motility associated with diabetes mellitus. Ann Intern Med 1983;98:378-384.

-2 Bytzer P, Talley NJ, Leemon M, Young LJ, Jones MP, Horowitz M: Prevalence of gastrointestinal symptoms associated with diabetes mellitus: a population-based survey of 15,000 adults. Arch Intern Med 2001;161: 1989-1996.

-3 Bytzer P, Talley NJ, Hammer J, Young LJ, Jones MP, Horowitz M: GI symptoms in diabetes mellitus are associated with both poor glycemic control and diabetic complications. Am J Gastroenterol 2002;97:604-611.

4 Shakil A, Church RJ, Rao SS: Gastrointestinal complications of diabetes. Am Fam Physician 2008;77: 1697-1702.

5 Ko GT, Chan WB, Chan JC, Tsang LW, Cockram CS: Gastrointestinal symptoms in Chinese patients with type 2 diabetes mellitus. Diabet Med 1999;16:670-674.

6 Dandona P, Fonseca V, Mier A, Beckett AG: Diarrhea and metformin in a diabetic clinic. Diabetes Care 1983;6:472-474.

7 Lysy J, Israeli E, Goldin E: The prevalence of chronic diarrhea among diabetic patients. Am J Gastroenterol 1999;94:2165-2170.

-8 Virally-Monod M, Tielmans D, Kevorkian JP, Bouhnik Y, Flourie B, Porokhov B, Ajzenberg C, Warnet A, Guillausseau PJ: Chronic diarrhoea and diabetes mellitus: prevalence of small intestinal bacterial overgrowth. Diabetes Metab 1998;24:530-536.

-9 Shakov R, Salazar RS, Kagunye SK, Baddoura WJ, Debari VA: Diabetes mellitus as a risk factor for recurrence of Clostridium difficile infection in the acute care hospital setting. Am J Infect Control 2011;39:194-198.

10 Spotnitz WD, Van Natta FC, Bashist B, Wolff M, Green P, Weber CJ: Localized ischemic colitis in a young woman with diabetes. Dis Colon Rectum 1984;27:481-484.

11 Sharieff GQ, Shad JA, Garmel G: An unusual case of mesenteric ischemia in a patient with new-onset diabetes mellitus. Am J Emerg Med 1997;15:282-284.

12 Nagai T, Tomizawa T, Monden T, Mori M: Diabetes mellitus accompanied by nonocclusive colonic ischemia. Intern Med 1998;37:454-456.

13 Medina C, Vilaseca J, Videla S, Fabra R, Armengol-Miro JR, Malagelada JR: Outcome of patients with ischemic colitis: review of fifty-three cases. Dis Colon Rectum 2004;47:180-184.

14 Longstreth GF, Yao JF: Diseases and drugs that increase risk of acute large bowel ischemia. Clin Gastroenterol Hepatol 2010;8:49-54.

15 Cubiella Fernández J, Núñez Calvo L, González Vázquez E, García García MJ, Alves Pérez MT, Martínez Silva I, Fernández Seara J: Risk factors associated with the development of ischemic colitis. World J Gastroenterol 2010;16:4564-4569. 


\begin{tabular}{c|l|l|l}
$\begin{array}{r}\text { Case Reports in } \\
\text { Gastroenterology }\end{array}$ & $\begin{array}{l}\text { Case Rep Gastroenterol 2011;5:239-245 } \\
\text { DOI: 10.1159/000327981 }\end{array}$ & $\begin{array}{l}\text { Published online: } \\
\text { April 15, 2011 }\end{array}$ & $\begin{array}{l}\text { O 2011 S. Karger AG, Basel } \\
\text { ISSN 1662-0631 } \\
\text { www.karger.com/crg }\end{array}$ \\
\hline
\end{tabular}

16 Kandemir O, Utas C, Gönen O, Patiroglu TE, Ozbakir O, Kelestimur F, Yücesoy M: Colonic subepithelial collagenous thickening in diabetic patients. Dis Colon Rectum 1995;38:1097-1100.

17 De Las Casas LE, Finley JL: Diabetic microangiopathy in the small bowel. Histopathology 1999;35:267-270.

18 Mitsudo S, Brandt LJ: Pathology of intestinal ischemia. Surg Clin North Am 1992;72:43-63.

19 Dignan CR, Greenson JK: Can ischemic colitis be differentiated from C. difficile colitis in biopsy specimens? Am J Surg Pathol 1997;21:706-710.

20 Robson NK, Khan SM, Rawlinson J, Dewbury KC: Ischaemic colitis: clinical, radiological and pathological correlation in three cases. Clin Radiol 1992;46:337-339. 